\title{
INERTIAL NORMAL S-TYPE TSENG'S EXTRAGRADIENT ALGORITHM FOR SOLUTION OF VARIATIONAL INEQUALITY PROBLEMS
}

\author{
D.R. Sahu And Amit Kumar Singh*®
}

\begin{abstract}
In this paper, we introduce inertial Tseng's extragradient algorithms combined with normal-S iteration process for solving variational inequality problems involving pseudo-monotone and Lipschitz continuous operators. Under mild conditions, we establish the weak convergence results in Hilbert spaces. Numerical examples are also presented to show that faster behaviour of the proposed method.
\end{abstract}

Mathematics Subject Classification. 47J05, 47J25, 47J20.

Received December 4, 2020. Accepted June 16, 2021.

\section{INTRODUCTION}

Variational inequality problems are extremely powerful tools to study the many nonlinear problems arising in several branches of applied science in different framework due to its successful applications in fields including control theory, game theory, transportation science, economic equilibrium and engineering sciences. It is worth mentioning that the variational inequality problem is a central problem in optimization theory. The variational inequality problem $\operatorname{VI}(C, A)$ is to find $p \in C$ such that

$$
\langle A p, x-p\rangle \geq 0 \text { for all } x \in C,
$$

where $C$ is a nonempty closed convex subset of Hilbert space $X$ with inner product $\langle\cdot, \cdot\rangle$ and the induced norm $\|\cdot\|$ and $A: X \rightarrow X$ is given nonlinear operator. It is a useful mathematical model which unifies many important concepts in applied mathematics, such as network equilibrium problems, necessary optimality conditions, systems of nonlinear equations and complementarity problems. Many research articles have been published to study and solve the variational inequality problem (1.1) (see, [6, 7, 9, 21, 28-30,39, 41, 42, 44]). Regularized and projection methods became very popular to solve variational inequality problem (1.1). In this paper, we focus on projection methods for solving the variational inequality problems, introduced by Goldstein [10] and defined by

$$
\left\{\begin{array}{l}
x_{1} \in C, \\
x_{n}=P_{C}\left(x_{n}-\lambda A x_{n}\right) \quad \text { for all } n \in \mathbb{N},
\end{array}\right.
$$

Keywords. Pseudo-Monotone mapping, Tseng's Extragradient Method, Variational Inequality Problems.

Department of Mathematics, Institute of Science, Banaras Hindu University, Varanasi 221005, India.

*Corresponding author: amit.bsingh1992@gmail.com 
for the convergence of the above method requires slightly strong assumption that operators are strongly monotone and Lipschitz continuous. Recently, authors in $[12,17-19,22]$ studied the convergence behaviour of projection methods and its variants for strongly pseudo-monotone and Lipschitzian variational inequality problems. It is worth mentioning that the class of strongly pseudomonotone variational inequality problems properly contains the class of strongly monotone variational inequality problems. It is noted that if we relax the strong monotonicity condition of an operator to monotonicity, then the sequence generated by projection method (1.2) may diverge. To deal with this drawback of method defined by (1.2), Korpelevich [20] introduced the extragradient method under the mild assumption of monotonicity of the operator. The extragradient method is given as follows:

$$
\left\{\begin{array}{l}
y_{n}=P_{C}\left(x_{n}-\lambda_{n} A x_{n}\right), \\
x_{n+1}=P_{C}\left(x_{n}-\lambda_{n} A y_{n}\right) \quad \text { for all } n \in \mathbb{N} .
\end{array}\right.
$$

The extragradient method, needs to calculate two projections onto the feasible set $C$ and two evaluation of $A$ in each iteration, it is well known that the projection onto a closed convex set $C$ is related to a minimum distance problem. So, if $C$ is a general closed and convex set, then a minimal distance problem has to be solved in order to obtain the next iterate. This might seriously affect the efficiency of the extragradient method, so the extragradient method needs to be improved in a situation, where a projection onto $C$ is hard to evaluate and therefore computationally expansive. To our knowledge, there are some kinds of methods to over this drawback. One of those, Tseng's extragradient method [36] introduced by Tseng's, which requires to calculate only one projection per iteration is given as follows:

$$
\left\{\begin{array}{l}
x_{1} \in C, \\
y_{n}=P_{C}\left(x_{n}-\lambda A x_{n}\right), \\
x_{n+1}=y_{n}-\lambda\left(A y_{n}-A x_{n}\right) \quad \text { for all } n \in \mathbb{N} .
\end{array}\right.
$$

The weak convergence of Tseng's extragradient method for solving monotone Lipschitz continuous variational inequalities was established in [36], with a different choice of parameters, Thong and Hieu [34] established the strong convergence results for strongly pseudomonotone variational inequalities. In 2018, Thong and Hieu [34] combined the inertial method with Tseng's extragradient algorithms (1.4) to introduce inertial Tseng's extragradient algorithms is given as follows:

$$
\left\{\begin{array}{l}
x_{0}, x_{1} \in X \\
z_{n}=x_{n}+\alpha_{n}\left(x_{n}-x_{n-1}\right), \\
y_{n}=P_{C}\left(z_{n}-\lambda A z_{n}\right), \\
x_{n+1}=y_{n}-\lambda\left(A y_{n}-A z_{n}\right) \quad \text { for all } n \in \mathbb{N}
\end{array}\right.
$$

for solving the monotone variational inequality problems with better performance under the suitable choice of parameters. In 2019, Thong and Vuong [35] proposed variants of Tseng's extragradient with a suitable linesearch to remove the dependency on the Lipschitz continuity modulus of operator $A$, and also reduced the Lipschitz continuity of $A$ to the uniform continuity. Very recently, Yao et al. [43] presented a variant of Tseng's extrgradient method to compute the common solution of variational inequality problem for monotone and Lipschitz continuous operator and fixed point for Lipschitz pseudocontractive operator. Tseng's extragradient method for solving variational inequality problems has received great attention by many authors (see, $[4,5,31-$ $33,38]$ and the references therein). Some important methods for class of pseudomonotone variational inequality problems can be found, for example, in [11,13-16]. In 2016, Yao et al. [40] constructed an implicit algorithm to solving the hierarchical monotone variational inequality.

In order to achieve the faster convergence speed of algorithm time dynamical system, Polyak [23] introduced the inertial method, which can be considered as a second order discretization of the dynamical system. The inertial algorithm is a two-step iterative method, and the next iterate is defined by making use of the previous two iterates and it can be regarded as a procedure of speeding up the convergence properties. 
Recently, a lot of researchers constructed fast iterative algorithms by using inertial extrapolation, including inertial forwardbackward splitting methods, inertial DouglasRachford splitting method, inertial ADMM, inertial forward-backward-forward method, inertial proximal-extragradient method, inertial contraction method, inertial subgradient extragradient method and inertial Mann iterative methods.

On the other hand, in 2011, Sahu [24] introduced the notion of S-operator of a mapping $T$ generated by $\alpha \in(0,1)$ and $T$ and normal S-iteration process as a special case of S-iteration process in the following way: Let $C$ be a nonempty convex subset of a normed linear space $X$ and $T: C \rightarrow C$ an operator. Then, for arbitrary $x_{1} \in C$, generate a sequence $\left\{x_{n}\right\}$ as

$$
x_{n+1}=T\left[\left(1-\alpha_{n}\right) x_{n}+\alpha_{n} T\left(x_{n}\right)\right], n \in \mathbb{N},
$$

where $\left\{\alpha_{n}\right\}$ is a sequence of real numbers in $(0,1)$. The rate of convergence of S-iteration process and normal S-iteration process is faster than the Picard and Mann iteration process for contraction mappings. From the literature, it is easy to observe that rate of convergence of S-iteration process and normal S-iteration process is faster than the Picard and Mann iteration process for contraction mappings. Recently, S-iterative methodology is applied for solving various nonlinear problems, inclusion problems, optimization problems etc. (see [25-27]). Inertial version of normal S-iteration process for nonexpansive mapping is studied by Verma and Shukla [37] and Dixit et al. [8].

In this article, motivated by the work of Tseng's [36] and Sahu [24], we consider the variational inequality problem in Hilbert spaces and proposed an inertial normal S-type Tseng's extragradient Algorithm for solution of variational inequality problems. Also, we prove the weak convergence of the sequences generated by the proposed algorithms.

This paper is organized as follows: Section 2 consists of the notations and basic definitions which is useful throughout the paper and also contains the examples and lemmas used to prove our main result. Section 3, we define the iterative algorithm in view of inertial normal S-type Tseng's extragradient Algorithm for solution of variational inequality problems in the framework of real Hilbert spaces. We also present some propositions and theorems to study the convergence behaviour of the proposed algorithm. In Section 4, we give a numerical example to demonstrate the convergence behaviour of the proposed algorithm.

\section{Preliminaries}

In this section, we collect some essential definitions, examples and lemmas, which will be further used in this paper. Let $C$ be a nonempty subset of a real Hilbert space $X$ with inner product $\langle\cdot, \cdot\rangle$ and norm $\|\cdot\|$, respectively. The strong (weak) convergence of a sequence $\left\{x_{n}\right\}$ to $x$ will be denoted by $x_{n} \rightarrow x\left(x_{n} \rightarrow x\right)$, respectively. For a given sequence $\left\{x_{n}\right\} \subset X, w\left(x_{n}\right)$ denote the weak $w$-limit set of $\left\{x_{n}\right\}$, that is,

$$
w_{w}\left(x_{n}\right):=\left\{x \in X: x_{n_{j}} \rightarrow x \text { for some subsequence }\left\{n_{j}\right\} \text { of }\{n\}\right\} .
$$

Definition $2.1([1])$. Let $C$ be a nonempty subset of a real Hilbert space $X$. An operator $A: C \rightarrow X$ is said to be

(1) monotone if

$$
\langle A x-A y, x-y\rangle \geq 0 \text { for all } x, y \in C,
$$

(2) $\beta$-strongly monotone if there exists a real number $\beta>0$ such that

$$
\langle A x-A y, x-y\rangle \geq \beta\|x-y\|^{2} \text { for all } x, y \in C,
$$

(3) $\alpha$-inverse strongly monotone if there exists a real number $\alpha>0$ such that

$$
\langle A x-A y, x-y\rangle \geq \alpha\|A x-A y\|^{2} \quad \text { for all } \quad x, y \in C,
$$


(4) $k$-Lipschitz continuous if there exists a real number $k>0$ such that

$$
\|A x-A y\| \leq k\|x-y\| \text { for all } x, y \in C,
$$

(5) nonexpansive if

$$
\|A x-A y\| \leq\|x-y\| \text { for all } x, y \in C,
$$

(6) pseudo-monotone if

$$
\langle A x, y-x\rangle \geq 0 \Rightarrow\langle A y, y-x\rangle \geq 0 \text { for all } x, y \in C,
$$

(7) sequential weak to weak continuous if for every sequence

$$
x_{n} \rightarrow x \Rightarrow A\left(x_{n}\right) \rightarrow A(x) .
$$

Let $C$ be a nonempty closed convex subset of a real Hilbert space $X$. For every element $x \in X$, there exists a unique nearest point in $C$, denoted by $P_{C} x$, such that

$$
\left\|x-P_{C} x\right\|=\inf \{\|x-y\|: y \in C\} .
$$

$P_{C}$ is called the metric projection of $X$ onto $C$. We know that $P_{C}$ is a nonexpansive operator.

Lemma 2.2 ([8]). Let $X$ be a real Hilbert space. Then, for $\rho>0$, and $\alpha \in \mathbb{R}$, we have

$$
\|x \pm \alpha y\|^{2} \geq(1-\alpha \rho)\|x\|^{2}+\alpha\left(\alpha-\frac{1}{\rho}\right)\|y\|^{2} \text { for all } x, y \in X .
$$

Lemma 2.3 ([1]). Let $C$ be a nonempty closed convex subset of a real Hilbert space $X$. Given $x \in X$ and $z \in C$. Then,

$$
P_{C}(x)=z \Leftrightarrow\langle x-z, y-z\rangle \leq 0 \text { for all } y \in C .
$$

Lemma 2.4 ([3] p. 30). Let $X$ be a real Hilbert space and let $t \in \mathbb{R}$. Then

$$
\|t x+(1-t) y\|^{2}=t\|x\|^{2}+(1-t)\|y\|^{2}-t(1-t)\|x-y\|^{2} \text { for all } x, y \in X .
$$

Lemma $2.5([2])$. Let $X$ be a real Hilbert space and $\left\{x_{n}\right\} \subset X$ such that there exists a nonempty, closed and convex subset $C$ of $X$ satisfying

(i) for every $z \in C, \lim _{n \rightarrow \infty}\left\|x_{n}-z\right\|$ exists;

(ii) any weak cluster point of $\left\{x_{n}\right\}$ belongs to $C$.

Then, there exists $x^{*} \in C$ such that $\left\{x_{n}\right\}$ converges weakly to $x^{*}$.

Lemma 2.6 ([32]). Assume that $A: C \rightarrow X$ is continuous and pseudo-monotone operator. Then, $x^{*}$ is a solution of (1.1) if and only if $x^{*}$ is a solution of the following problem:

$$
\text { find } x \in C \text { such that }\langle A y, y-x\rangle \geq 0 \text { for all } y \in C \text {. }
$$

Lemma $2.7([2])$. Let $\left\{a_{n}\right\},\left\{b_{n}\right\}$ and $\left\{c_{n}\right\}$ be the sequences in $[0, \infty)$ such that

$$
a_{n+1} \leq a_{n}+c_{n}\left(a_{n}-a_{n-1}\right)+b_{n} \text { for all } n \in \mathbb{N}, \quad \sum_{n=1}^{\infty} b_{n}<\infty
$$

and there exists a real number $c$ with $0 \leq c_{n} \leq c<1$ for all $n \in \mathbb{N}$. Then the following hold:

(i) $\sum_{n=1}^{\infty}\left[a_{n}-a_{n-1}\right]_{+}<\infty$, where $[r]_{+}=\max \{r, 0\}$,

(ii) there exists $a^{*} \in[0, \infty)$ such that $a_{n} \rightarrow a^{*}$. 


\section{MAin Results}

In this section, we propose an iterative algorithm in view of Tseng's extragradient method and normal-S iteration process for solution of variational inequality problem (1.1) for pseudo-monotone Lipschitz operator in the setting of Hilbert spaces.

Algorithm 3.1. Let $C$ be a nonempty closed convex subset of real Hilbert space $X$ and $A: X \rightarrow X$ be a pseudo-monotone and $L$-Lipschitz continuous operator. Let $\mu \in(0,1 / L)$ and let $\left\{\alpha_{n}\right\}$ be a sequence of real number in $[0,1)$ and $\left\{\beta_{n}\right\}$ a sequence of real number in $(0,1)$. Define a sequence $\left\{x_{n}\right\}$ in $X$ by

$$
\left\{\begin{array}{l}
x_{0}, x_{1} \in X \\
z_{n}=x_{n}+\alpha_{n}\left(x_{n}-x_{n-1}\right), \\
u_{n}=P_{C}\left(z_{n}-\mu A z_{n}\right), \\
y_{n}=\left(1-\beta_{n}\right) z_{n}+\beta_{n}\left[u_{n}-\mu\left(A u_{n}-A z_{n}\right)\right], \\
w_{n}=P_{C}\left(y_{n}-\mu A y_{n}\right), \quad \text { for all } n \in \mathbb{N} . \\
x_{n+1}=w_{n}-\mu\left(A w_{n}-A y_{n}\right) \quad
\end{array}\right.
$$

We assume that $\left\{\alpha_{n}\right\}$ and $\left\{\beta_{n}\right\}$ satisfy the following conditions:

(D1) $\left\{\alpha_{n}\right\} \subset[0, \alpha]$ is non-decreasing sequence with $\alpha \in[0,1)$;

(D2) constants $\beta, \sigma, \delta>0$ satisfying

$$
\begin{aligned}
& \delta>\frac{2 q \alpha L^{* 2}(\alpha(1+\alpha)+\sigma)}{1-\alpha^{2}(1-\beta)}, \\
& 0<\beta \leq \beta_{n} \leq \frac{\delta-\alpha\left(2 q L^{*^{2}} \alpha(1+\alpha)+\alpha \delta(1-\beta)+2 q L^{* 2} \sigma\right)}{\delta\left[1+2 q L^{* 2} \alpha(1+\alpha)+\alpha \delta(1-\beta)+2 q L^{* 2} \sigma\right]},
\end{aligned}
$$

where $q=1+\frac{1}{\beta^{2}}$

(D3) define sequences $\left\{\xi_{n}\right\}$ and $\left\{\mu_{n}\right\}$ by

$$
\begin{aligned}
& \mu_{n}=\alpha_{n}\left(1+\alpha_{n}\right)+\frac{\alpha_{n}\left(1-\beta_{n}\right)\left(1-\alpha_{n} \rho_{n}\right)}{2 q \beta_{n} L^{* 2} \rho_{n}}, \\
& \xi_{n}=-\frac{\left(1-\beta_{n}\right)\left(1-\alpha_{n} \rho_{n}\right)}{2 q \beta_{n} L^{* 2}},
\end{aligned}
$$

where $L^{*}=(1+\mu L)^{2}+\mu L$ and $\rho_{n}=\frac{1}{\alpha_{n}+\delta \beta_{n}}$.

- If we consider $\alpha_{n}=0$ for all $n \in \mathbb{N}$, then Algorithm 3.1 reduces to the following algorithm:

$$
\left\{\begin{array}{l}
x_{1} \in C, \\
u_{n}=P_{C}\left(x_{n}-\mu A x_{n}\right), \\
y_{n}=\left(1-\beta_{n}\right) x_{n}+\beta_{n}\left[u_{n}-\mu\left(A u_{n}-A x_{n}\right)\right], \\
w_{n}=P_{C}\left(y_{n}-\mu A y_{n}\right), \\
x_{n+1}=w_{n}-\mu\left(A w_{n}-A y_{n}\right), \quad \text { for all } n \in \mathbb{N} .
\end{array}\right.
$$

Thus, (3.4) is normal S-iteration based Tseng's extragradient method (nS-TEM).

- If we consider operator $T: X \rightarrow X$ as $T=P_{C}(I-\mu A)-\mu\left(A P_{C}(I-\mu A)-A\right)$, then Algorithm 3.1 reduces to the following algorithm:

$$
\left\{\begin{array}{l}
x_{0}, x_{1} \in X \\
z_{n}=x_{n}+\alpha_{n}\left(x_{n}-x_{n-1}\right), \\
y_{n}=\left(1-\beta_{n}\right) z_{n}+\beta_{n} T z_{n}, \\
x_{n+1}=T y_{n} \quad \text { for all } n \in \mathbb{N} .
\end{array}\right.
$$


Thus, (3.1) is inertial normal S-iteration based Tseng's extragradient method (InS-TEM).

In order to study the convergence analysis of InS-TEM (3.1), we establish the following propositions.

Proposition 3.2. Let $C$ be a nonempty closed convex subset of a real Hilbert space $X$ and $A: X \rightarrow X$ be a pseudo-monotone Lipschitz operators with Lipschitz constant L. Define an operator $T: X \rightarrow X$ such that

$$
T=P_{C}(I-\mu A)-\mu\left(A P_{C}(I-\mu A)-A\right)
$$

where $\mu \in(0,1 / L)$. Then we have the following:

(a) $T$ is Lipschitz mapping with Lipschitz constant $\left((1+\mu L)^{2}+\mu L\right)$.

(b) $\|T z-p\|^{2} \leq\|z-p\|^{2}-\left(1-\mu^{2} L^{2}\right)\|u-z\|^{2}$.

(c) If $\mu \in\left(0, \frac{1}{L}\right)$ then $T$ is quasi-nonexpansive operator.

Proof. (a) Let $x, y \in X$. Then

$$
\begin{aligned}
\|T x-T y\| & =\left\|\left[P_{C}(I-\mu A)-\mu\left(A P_{C}(I-\mu A)-A\right)\right] x-\left[P_{C}(I-\mu A)-\mu\left(A P_{C}(I-\mu A)-A\right)\right] y\right\| \\
& \leq\left\|P_{C}(I-\mu A) x-P_{C}(I-\mu A) y\right\|+\mu\left\|\left[\left(A P_{C}(I-\mu A)-A\right)\right] x-\left[\left(A P_{C}(I-\mu A)-A\right)\right] y\right\| \\
& \leq\left\|P_{C}(I-\mu A) x-P_{C}(I-\mu A) y\right\|+\mu\left\|A\left(P_{C}(I-\mu A)\right) x-A\left(P_{C}(I-\mu A)\right) y\right\|+\mu\|A x-A y\| \\
& \leq\left\|P_{C}(I-\mu A) x-P_{C}(I-\mu A) y\right\|+\mu L\left\|P_{C}(I-\mu A) x-P_{C}(I-\mu A) y\right\|+\mu L\|x-y\| \\
& =(1+\mu L)\left\|P_{C}(I-\mu A) x-P_{C}(I-\mu A) y\right\|+\mu L\|x-y\| \\
& \leq(1+\mu L)\|(x-y)-\mu(A x-A y)\|+\mu L\|x-y\| \\
& \leq(1+\mu L)\{\|x-y\|+\mu\|(A x-A y)\|\}+\mu L\|x-y\| \\
& \leq(1+\mu L)^{2}\|x-y\|+\mu L\|x-y\| \\
& =\left((1+\mu L)^{2}+\mu L\right)\|x-y\| \\
& =L^{*}\|x-y\|,
\end{aligned}
$$

where $L^{*}=(1+\mu L)^{2}+\mu L$. Therefore, $T: C \rightarrow X$ is $L^{*}$-Lipschitz continuous operator.

(b) Let $p \in \Omega[\operatorname{VI}(C, A)]$. Set $u=P_{C}(I-\mu A) z$, therefore

$$
\begin{aligned}
\|T z-p\|^{2}= & \|u-\mu(A u-A z)-p\|^{2} \\
= & \|u-p-\mu(A u-A z)\|^{2} \\
= & \|u-p\|^{2}+\mu^{2}\|A u-A y\|^{2}-2 \mu\langle u-p, A u-A z\rangle \\
= & \|u-z+z-p\|^{2}+\mu^{2}\|A u-A z\|^{2}-2 \mu\langle u-p, A u-A z\rangle \\
= & \|z-p\|^{2}+\|u-z\|^{2}+2\langle u-z, z-p\rangle \\
& +\mu^{2}\|A u-A z\|^{2}-2 \mu\langle u-p, A u-A z\rangle \\
= & \|z-p\|^{2}+\|u-z\|^{2}+2\langle u-z, z-u+u-p\rangle \\
& +\mu^{2}\|A u-A z\|^{2}-2 \mu\langle u-p, A u-A z\rangle \\
= & \|z-p\|^{2}+\|u-z\|^{2}-2\langle u-z, u-z\rangle+2\langle u-z, u-p\rangle \\
& +\mu^{2}\|A u-A z\|^{2}-2 \mu\langle u-p, A u-A z\rangle \\
\leq & \|z-p\|^{2}-\|u-z\|^{2}+2\langle u-z, u-p\rangle \\
& +\mu^{2} L^{2}\|u-z\|^{2}-2 \mu\langle u-p, A u-A z\rangle \\
= & \|z-p\|^{2}-\left(1-\mu^{2} L^{2}\right)\|u-z\|^{2}+2\langle u-z, u-p\rangle \\
& -2 \mu\langle u-p, A u-A z\rangle .
\end{aligned}
$$


Since $u=P_{C}(z-\mu A z)$, then from Lemma 2.3, we obtain

$$
\langle u-z+\mu A z, u-p\rangle \leq 0 \text { for all } p \in C,
$$

which implies that

$$
\langle u-z, u-p\rangle \leq-\mu\langle A z, u-p\rangle \text { for all } p \in C .
$$

From (3.7), we have

$$
\begin{aligned}
\|T z-p\|^{2} \leq & \|z-p\|^{2}-\left(1-\mu^{2} L^{2}\right)\|u-z\|^{2}-2 \mu\langle A z, u-p\rangle \\
& -2 \mu\langle u-p, A u-A z\rangle \\
= & \|z-p\|^{2}-\left(1-\mu^{2} L^{2}\right)\|u-z\|^{2}-2 \mu\langle u-p, A u\rangle .
\end{aligned}
$$

Since, $p \in \Omega[\operatorname{VI}(C, A)]$, we have $\langle A p, u-p\rangle \geq 0$ for all $z \in C$. By pseudomonotonocity of $A$, we have

$$
\langle A u, u-p\rangle \geq 0 \text { for all } p \in C .
$$

From (3.8), we have

$$
\|T z-p\|^{2} \leq\|z-p\|^{2}-\left(1-\mu^{2} L^{2}\right)\|u-z\|^{2} .
$$

(c) Since $\mu \in(0,1 / L)$, we obtain from (3.9)

$$
\|T z-p\| \leq\|z-p\|
$$

Hence $T$ is quasi-nonexpansive operator.

Proposition 3.3. Let $C$ be a nonempty closed convex subset of a real Hilbert space $X$ and $A: X \rightarrow X$ be a pseudomonotone and L-Lipschitz continuous operator such that $T$ is sequentially weak to weak continuous and $\Omega[\operatorname{VI}(C, A)] \neq \emptyset$. Let $\left\{x_{n}\right\}$ be a bounded sequence in $X$ and $y_{n}=P_{C}\left(x_{n}-\lambda A x_{n}\right)$ such that $\left\|x_{n}-y_{n}\right\| \rightarrow 0$. Then $\left\langle A(x), x-x^{*}\right\rangle \geq 0$ for $x \in C$ and $x^{*} \in \Omega[\operatorname{VI}(C, A)]$.

Proof. Let $x \in C$ and $x^{*} \in \Omega[\operatorname{VI}(C, A)]$. Since the sequence $\left\{x_{n}\right\}$ is bounded, we assume that there exists a subsequence $\left\{x_{n_{k}}\right\}$ of $\left\{x_{n}\right\}$ such that $x_{n_{k}} \rightarrow x^{*}$. Note $y_{n_{k}}=P_{C}\left(x_{n_{k}}-\lambda A x_{n_{k}}\right)$, we have

$$
\left\langle x_{n_{k}}-\lambda A x_{n_{k}}-y_{n_{k}}, y_{n_{k}}-x\right\rangle \geq 0,
$$

which implies that

$$
\begin{aligned}
0 & \leq\left\langle y_{n_{k}}-x_{n_{k}}+\lambda A x_{n_{k}}, x-y_{n_{k}}\right\rangle \\
& =\left\langle y_{n_{k}}-x_{n_{k}}, x-y_{n_{k}}\right\rangle+\lambda\left\langle A x_{n_{k}}, x-y_{n_{k}}\right\rangle \\
& =\left\langle y_{n_{k}}-x_{n_{k}}, x-y_{n_{k}}\right\rangle+\lambda\left\langle A x_{n_{k}}-A y_{n_{k}}, x-y_{n_{k}}\right\rangle+\lambda\left\langle A y_{n_{k}}, x-x_{n_{k}}\right\rangle \\
& \leq\left\|y_{n_{k}}-x_{n_{k}}\right\|\left\|x-y_{n_{k}}\right\|+\lambda L\left\|y_{n_{k}}-x_{n_{k}}\right\|\left\|x-y_{n_{k}}\right\|+\lambda\left\langle A y_{n_{k}}, x-x_{n_{k}}\right\rangle .
\end{aligned}
$$

Note that $\lim _{k \rightarrow \infty}\left\|x_{n_{k}}-y_{n_{k}}\right\|=0$ and $\left\{y_{n_{k}}\right\}$ is bounded, it follows

$$
\liminf _{k \rightarrow \infty}\left\langle A y_{n_{k}}, x-x_{n_{k}}\right\rangle \geq 0 .
$$

Let $\left\{\epsilon_{k}\right\}$ be a positive strictly decreasing sequence which converges to 0 as $k \rightarrow \infty$. We can construct inductively a strictly increasing sequence $\left\{N_{k}\right\}$ with the property that

$$
\left\langle A y_{n_{N_{k}}}, x-y_{n_{N_{k}}}\right\rangle+\epsilon_{k} \geq 0 \text { for all } k \geq 0 .
$$


For every $k \geq 0$ we have $A y_{n_{N_{k}}} \neq 0$ and setting

$$
z_{k}=\frac{A y_{n_{N_{k}}}}{\left\|A y_{n_{N_{k}}}\right\|^{2}},
$$

it holds $\left\langle A y_{n_{N_{k}}}, z_{k}\right\rangle=1$. From (3.12), we have

$$
\begin{aligned}
\left\langle A y_{n_{N_{k}}}, x+\epsilon_{k} z_{k}-y_{n_{N_{k}}}\right\rangle & =\left\langle A y_{n_{N_{k}}}, x-y_{n_{N_{k}}}\right\rangle+\epsilon_{k}\left\langle A y_{n_{N_{k}}}, z_{k}\right\rangle \\
& =\left\langle A y_{n_{N_{k}}}, x-y_{n_{N_{k}}}\right\rangle+\epsilon_{k} \geq 0 \quad \text { for all } k \geq 0 .
\end{aligned}
$$

Since $A$ is pseudo-monotone on $C$, this yields

$$
\left\langle A\left(x+\epsilon_{k} z_{k}\right), x+\epsilon_{k} z_{k}-y_{n_{N_{k}}}\right\rangle \geq 0 \text { for all } k \geq 0 .
$$

On the other hand, we have that $\left\{y_{n_{N_{k}}}\right\}$ converges weakly to $x^{*}$ as $k \rightarrow \infty$. Since $A$ is sequentially weak to weak continuous, $\left\{A\left(y_{n_{N_{k}}}\right)\right\}$ converges weakly to $A\left(x^{*}\right)$ as $k \rightarrow \infty$. Since the norm mapping is sequentially weakly lower semicontinuous, we have

$$
0<\left\|A\left(x^{*}\right)\right\| \leq \liminf _{k \rightarrow \infty}\left\|A\left(y_{n_{N_{k}}}\right)\right\|,
$$

which implies that $\left(\frac{1}{A\left(y_{n_{N}}\right)}\right)$ is bounded. Thus, we have

$$
\lim _{k \rightarrow \infty}\left\|\epsilon_{k} z_{k}\right\|=\lim _{k \rightarrow \infty} \frac{\epsilon_{k}}{\left\|A\left(y_{n_{N_{k}}}\right)\right\|}=0 .
$$

Taking limit as $k \rightarrow \infty$ in (3.14), we obtain

$$
\left\langle A(x), x-x^{*}\right\rangle \geq 0
$$

Now, we are ready to present convergence analysis of inertial normal S-iteration based Tseng's extragradient method (3.1).

Theorem 3.4. Let $C$ be a nonempty closed convex subset of a real Hilbert space $X$ and $A: X \rightarrow X$ be a pseudomonotone and L-Lipschitz continuous operator such that $T$ is sequentially weak to weak continuous and $\Omega[\operatorname{VI}(C, A)] \neq \emptyset$. Let $\left\{x_{n}\right\}$ be a sequence in $X$ generated from $x_{0}, x_{1} \in C$ and defined by Algorithm 3.1, where $\left\{\alpha_{n}\right\}$ and $\left\{\beta_{n}\right\}$ are sequences of positive real numbers satisfying the conditions $(D 1)$ and $(D 2)$. Then $\left\{x_{n}\right\}$ converges weakly to an element of $\Omega[\mathrm{VI}(C, A)]$.

Proof. Let $p \in \Omega[\operatorname{VI}(C, A)]$. From Proposition 3.2, we have

$$
\left\|T z_{n}-p\right\|^{2} \leq\left\|z_{n}-p\right\|^{2}-\left(1-\lambda^{2} L^{2}\right)\left\|u_{n}-z_{n}\right\|^{2} .
$$

Note that

$$
\begin{aligned}
\left\|y_{n}-p\right\|^{2} & =\left\|\left(1-\beta_{n}\right) z_{n}+\beta_{n} T z_{n}-p\right\|^{2} \\
& =\left\|\left(1-\beta_{n}\right)\left(z_{n}-p\right)+\beta_{n}\left(T z_{n}-p\right)\right\|^{2} \\
& =\left(1-\beta_{n}\right)\left\|z_{n}-p\right\|^{2}+\beta_{n}\left\|T z_{n}-p\right\|^{2}-\beta_{n}\left(1-\beta_{n}\right)\left\|z_{n}-T z_{n}\right\|^{2}
\end{aligned}
$$




$$
\leq\left\|z_{n}-p\right\|^{2}-\beta_{n}\left(1-\mu^{2} L^{2}\right)\left\|u_{n}-z_{n}\right\|^{2}-\beta_{n}\left(1-\beta_{n}\right)\left\|z_{n}-T z_{n}\right\|^{2} .
$$

From Algorithm 3.1, we have

$$
\begin{aligned}
\left\|x_{n+1}-p\right\|^{2}= & \left\|T y_{n}-p\right\|^{2} \\
\leq & \left\|y_{n}-p\right\|^{2}-\left(1-\mu^{2} L^{2}\right)\left\|w_{n}-y_{n}\right\|^{2} \\
\leq & \left\|z_{n}-p\right\|^{2}-\beta_{n}\left(1-\mu^{2} L^{2}\right)\left\|u_{n}-z_{n}\right\|^{2}-\left(1-\mu^{2} L^{2}\right)\left\|w_{n}-y_{n}\right\|^{2} \\
& -\beta_{n}\left(1-\beta_{n}\right)\left\|z_{n}-T z_{n}\right\|^{2} .
\end{aligned}
$$

From Algorithm 3.1, we have

$$
\begin{aligned}
\left\|T z_{n}-z_{n}\right\| & \leq\left\|T z_{n}-u_{n}\right\|+\left\|u_{n}-z_{n}\right\| \\
& =\mu\left\|A u_{n}-A z_{n}\right\|+\left\|u_{n}-z_{n}\right\| \\
& \leq(1+\mu L)\left\|u_{n}-z_{n}\right\|,
\end{aligned}
$$

which implies that

$$
\left\|u_{n}-z_{n}\right\| \geq \frac{1}{(1+\mu L)}\left\|T z_{n}-z_{n}\right\|
$$

Similarly,

$$
\begin{aligned}
\left\|T y_{n}-y_{n}\right\| & \leq\left\|T y_{n}-w_{n}\right\|+\left\|w_{n}-z_{n}\right\| \\
& =\mu\left\|A y_{n}-A w_{n}\right\|+\left\|w_{n}-y_{n}\right\| \\
& \leq(1+\mu L)\left\|y_{n}-w_{n}\right\|,
\end{aligned}
$$

which implies that

$$
\left\|y_{n}-w_{n}\right\| \geq \frac{1}{(1+\mu L)}\left\|T y_{n}-y_{n}\right\|
$$

From (3.18), we have

$$
\begin{aligned}
\left\|x_{n+1}-p\right\|^{2} & \leq\left\|z_{n}-p\right\|^{2}-\beta_{n} \frac{(1-\mu L)}{(1+\mu L)}\left\|T z_{n}-z_{n}\right\|^{2}-\frac{(1-\mu L)}{(1+\mu L)}\left\|T y_{n}-y_{n}\right\|^{2}-\beta_{n}\left(1-\beta_{n}\right)\left\|z_{n}-T z_{n}\right\|^{2} \\
& \leq\left\|z_{n}-p\right\|^{2}-\beta_{n}\left(1-\beta_{n}\right)\left\|z_{n}-T z_{n}\right\|^{2} .
\end{aligned}
$$

From Algorithm 3.1, we have

$$
\begin{aligned}
\left\|z_{n}-T z_{n}\right\|^{2} & =\frac{1}{\beta_{n}^{2}}\left\|y_{n}-z_{n}\right\|^{2} \\
& =\frac{L^{* 2}}{\beta_{n}^{2} L^{* 2}}\left\|y_{n}-z_{n}\right\|^{2} \\
& \geq \frac{1}{\beta_{n}^{2} L^{* 2}}\left\|T y_{n}-T z_{n}\right\|^{2} \\
& =\frac{1}{\beta_{n}^{2} L^{* 2}}\left\|x_{n+1}-T z_{n}\right\|^{2} \\
& =\frac{1}{\beta_{n}^{2} L^{* 2}}\left\|x_{n+1}-z_{n}+z_{n}-T z_{n}\right\|^{2}
\end{aligned}
$$




$$
=\frac{1}{\beta_{n}^{2} L^{*^{2}}}\left\{\frac{1}{2}\left\|x_{n+1}-z_{n}\right\|^{2}+\left\|z_{n}-T z_{n}\right\|^{2}\right\},
$$

which implies that

$$
\left(1+\frac{1}{\beta_{n}^{2} L^{* 2}}\right)\left\|z_{n}-T z_{n}\right\|^{2} \geq \frac{1}{2 \beta_{n}^{2} L^{*^{2}}}\left\|x_{n+1}-z_{n}\right\|^{2} .
$$

Note $0<\beta \leq \beta_{n}$ for all $n \in \mathbb{N}$. Hence

$$
\begin{aligned}
\left(1+\frac{1}{\beta^{2} L^{* 2}}\right)\left\|z_{n}-T\left(z_{n}\right)\right\|^{2} & \geq \frac{1}{2 \beta_{n}^{2} L^{* 2}}\left\|x_{n+1}-z_{n}\right\|^{2} \\
& =\frac{1}{2 \beta_{n}^{2}}\left\|x_{n+1}-x_{n}-\alpha_{n}\left(x_{n}-x_{n-1}\right)\right\|^{2} .
\end{aligned}
$$

Again using Lemma 2.2, we obtain

$$
\begin{aligned}
\left(1+\frac{1}{\beta^{2} L^{* 2}}\right)\left\|z_{n}-T\left(z_{n}\right)\right\|^{2} & \geq \frac{\left(1-\alpha_{n} \rho_{n}\right)}{2 \beta_{n}^{2} L^{* 2}}\left\|x_{n+1}-x_{n}\right\|^{2}+\frac{\alpha_{n}}{2 \beta_{n}^{2} L^{* 2}}\left(\alpha_{n}-\frac{1}{\rho_{n}}\right)\left\|x_{n}-x_{n-1}\right\|^{2} \\
& =\frac{\left(1-\alpha_{n} \rho_{n}\right)}{2 \beta_{n}^{2} L^{* 2}}\left\|x_{n+1}-x_{n}\right\|^{2}-\frac{\alpha_{n}\left(1-\alpha_{n} \rho_{n}\right)}{2 \beta_{n}^{2} L^{* 2} \rho_{n}}\left\|x_{n}-x_{n-1}\right\|^{2} .
\end{aligned}
$$

Let $q=\left(1+\frac{1}{\beta^{2} L^{* 2}}\right)$ and multiplying $(3.21)$ by $-\beta_{n}\left(1-\beta_{n}\right)$, we obtain

$$
\begin{aligned}
-q \beta_{n}\left(1-\beta_{n}\right)\left\|z_{n}-T\left(z_{n}\right)\right\|^{2} \leq & -\frac{\left(1-\beta_{n}\right)\left(1-\alpha_{n} \rho_{n}\right)}{2 \beta_{n} L^{* 2}}\left\|x_{n+1}-x_{n}\right\|^{2} \\
& +\frac{\alpha_{n}\left(1-\beta_{n}\right)\left(1-\alpha_{n} \rho_{n}\right)}{2 \beta_{n} L^{* 2} \rho_{n}}\left\|x_{n}-x_{n-1}\right\|^{2} .
\end{aligned}
$$

and

$$
\begin{aligned}
\left\|z_{n}-p\right\|^{2} & =\left\|\left(1+\alpha_{n}\right)\left(x_{n}-p\right)-\alpha_{n}\left(x_{n-1}-p\right)\right\|^{2} \\
& =\left(1+\alpha_{n}\right)\left\|x_{n}-p\right\|^{2}-\alpha_{n}\left\|x_{n-1}-p\right\|^{2}+\alpha_{n}\left(1+\alpha_{n}\right)\left\|x_{n}-x_{n-1}\right\|^{2} .
\end{aligned}
$$

From Proposition 3.19 and (3.22), we get

$$
\begin{aligned}
\left\|x_{n+1}-p\right\|^{2} \leq & \left(1+\alpha_{n}\right)\left\|x_{n}-p\right\|^{2}-\alpha_{n}\left\|x_{n-1}-p\right\|^{2}+\alpha_{n}\left(1+\alpha_{n}\right)\left\|x_{n}-x_{n-1}\right\|^{2} \\
& -\frac{\left(1-\beta_{n}\right)\left(1-\alpha_{n} \rho_{n}\right)}{2 q \beta_{n} L^{* 2}}\left\|x_{n+1}-x_{n}\right\|^{2}+\frac{\alpha_{n}\left(1-\beta_{n}\right)\left(1-\alpha_{n} \rho_{n}\right)}{2 q \beta_{n} L^{* 2} \rho_{n}}\left\|x_{n}-x_{n-1}\right\|^{2} .
\end{aligned}
$$

Hence

$$
\begin{aligned}
\left\|x_{n+1}-p\right\|^{2}-\left(1+\alpha_{n}\right)\left\|x_{n}-p\right\|^{2}-\alpha_{n}\left\|x_{n-1}-p\right\|^{2} \leq & -\frac{\left(1-\beta_{n}\right)\left(1-\alpha_{n} \rho_{n}\right)}{2 q \beta_{n} L^{* 2}}\left\|x_{n+1}-x_{n}\right\|^{2} \\
& +\left\{\alpha_{n}\left(1+\alpha_{n}\right)+\frac{\alpha_{n}\left(1-\beta_{n}\right)\left(1-\alpha_{n} \rho_{n}\right)}{2 q \beta_{n} L^{* 2} \rho_{n}}\right\}\left\|x_{n}-x_{n-1}\right\|^{2},
\end{aligned}
$$

which can be written as

$$
\left\|x_{n+1}-p\right\|^{2}-\left(1+\alpha_{n}\right)\left\|x_{n}-p\right\|^{2}-\alpha_{n}\left\|x_{n-1}-p\right\|^{2} \leq \xi_{n}\left\|x_{n+1}-x_{n}\right\|^{2}+\mu_{n}\left\|x_{n}-x_{n-1}\right\|^{2},
$$


where $\xi_{n}=-\frac{\left(1-\beta_{n}\right)\left(1-\alpha_{n} \rho_{n}\right)}{2 q \beta_{n} L^{* 2}}$ and $\mu_{n}=\alpha_{n}\left(1+\alpha_{n}\right)+\frac{\alpha_{n}\left(1-\beta_{n}\right)\left(1-\alpha_{n} \rho_{n}\right)}{2 q \beta_{n} L^{* 2} \rho_{n}}$.

Let $\Gamma_{n}=\left\|x_{n}-p\right\|^{2}-\alpha_{n}\left\|x_{n-1}-p\right\|^{2}+\mu_{n}\left\|x_{n}-x_{n-1}\right\|^{2}$. Thus, we have

$$
\begin{aligned}
\Gamma_{n+1}-\Gamma_{n}= & \left\|x_{n+1}-p\right\|^{2}-\left(1+\alpha_{n}\right)\left\|x_{n}-p\right\|^{2}+\alpha_{n}\left\|x_{n-1}-p\right\|^{2} \\
& +\mu_{n+1}\left\|x_{n+1}-x_{n}\right\|^{2}-\mu_{n}\left\|x_{n}-x_{n-1}\right\|^{2} \\
\leq & \left(\xi_{n}+\mu_{n+1}\right)\left\|x_{n+1}-x_{n}\right\|^{2} .
\end{aligned}
$$

Note that $\mu_{n}=\alpha_{n}\left(1+\alpha_{n}\right)+\frac{\alpha_{n}\left(1-\beta_{n}\right)\left(1-\alpha_{n} \rho_{n}\right)}{2 q \beta_{n} L^{* 2} \rho_{n}}>0$, since $\alpha_{n} \rho_{n}<1$ and $\beta_{n} \in(0,1)$. Again, taking into account of choice of $\rho_{n}$, we have

$$
\delta=\frac{1-\alpha_{n} \rho_{n}}{\rho_{n} \beta_{n}} .
$$

Note

$$
\begin{aligned}
\mu_{n} & =\alpha_{n}\left(1+\alpha_{n}\right)+\frac{\alpha_{n}\left(1-\beta_{n}\right) \delta}{2 q L^{* 2}} \\
& \leq \alpha(1+\alpha)+\frac{\alpha \delta(1-\beta)}{2 q L^{* 2}} \text { for all } n \in \mathbb{N} .
\end{aligned}
$$

For $n \in \mathbb{N}$, we have

$$
\begin{aligned}
\xi_{n}+\mu_{n+1} \leq-\sigma & \Leftrightarrow \frac{\left(1-\beta_{n}\right)\left(\alpha_{n} \rho_{n}-1\right)}{2 q L^{* 2} \beta_{n}}+\left(\mu_{n+1}+\sigma\right) \leq 0 \\
& \Leftrightarrow\left(1-\beta_{n}\right)\left(\alpha_{n} \rho_{n}-1\right)+2 q \beta_{n} L^{* 2}\left(\mu_{n+1}+\sigma\right) \leq 0 \\
& \Leftrightarrow-\left(1-\beta_{n}\right) \delta \rho_{n} \beta_{n}+2 q \beta_{n} L^{* 2}\left(\mu_{n+1}+\sigma\right) \leq 0 \\
& \Leftrightarrow-\frac{\left(1-\beta_{n}\right) \delta}{\alpha_{n}+\delta \beta_{n}}+2 q L^{* 2}\left(\mu_{n+1}+\sigma\right) \leq 0 \\
& \Leftrightarrow-\left(1-\beta_{n}\right) \delta+2 q L^{* 2}\left(\mu_{n+1}+\sigma\right)\left(\alpha_{n}+\delta \beta_{n}\right) \leq 0 \\
& \Leftrightarrow 2 q L^{* 2}\left(\mu_{n+1}+\sigma\right)\left(\alpha_{n}+\delta \beta_{n}\right)+\beta_{n} \delta \leq \delta .
\end{aligned}
$$

By using (3.28), we have

$$
2 q L^{* 2}\left(\mu_{n+1}+\sigma\right)\left(\alpha_{n}+\delta \beta_{n}\right)+\beta_{n} \delta \leq 2 q L^{* 2}\left(\alpha(1+\alpha)+\frac{\alpha \delta(1-\beta)}{2 q}+\sigma\right)\left(\alpha+\delta \beta_{n}\right)+\beta_{n} \delta \leq \delta,
$$

where the last inequality follows by using the upper bound for $\left\{\beta_{n}\right\}$ in (3.2). Hence

$$
\xi_{n}+\mu_{n+1} \leq-\sigma \text { for all } n \in \mathbb{N} .
$$

This implies that

$$
\begin{aligned}
& \Gamma_{n+1}-\Gamma_{n} \leq-\delta\left\|x_{n+1}-x_{n}\right\|^{2} . \\
& \Gamma_{n+1}-\Gamma_{n} \leq 0 \quad \text { for all } n \geq \mathbb{N}_{0} .
\end{aligned}
$$

So, sequence $\left\{\Gamma_{n}\right\}$ is non-increasing, on the other hand, we have

$$
\begin{aligned}
\Gamma_{n} & =\left\|x_{n}-p\right\|^{2}-\alpha_{n}\left\|x_{n-1}-p\right\|^{2}+\mu_{n}\left\|x_{n}-x_{n-1}\right\|^{2} \\
& \geq\left\|x_{n}-p\right\|^{2}-\alpha_{n}\left\|x_{n+1}-p\right\|^{2} .
\end{aligned}
$$


This implies that

$$
\begin{aligned}
\left\|x_{n}-p\right\|^{2} & \leq \alpha_{n}\left\|x_{n-1}-p\right\|^{2}+\Gamma_{n} \\
& \leq \alpha\left\|x_{n-1}-p\right\|^{2}+\Gamma_{n_{0}} \\
& \vdots \\
& \leq \alpha^{n-n_{0}}\left\|x_{n_{0}}-p\right\|^{2}+\frac{\Gamma_{n_{0}}}{1-\alpha} .
\end{aligned}
$$

Thus, we have

$$
\begin{aligned}
\Gamma_{n+1} & =\left\|x_{n+1}-p\right\|^{2}-\alpha_{n+1}\left\|x_{n}-p\right\|^{2}+\mu_{n+1}\left\|x_{n+1}-x_{n}\right\|^{2} \\
& \geq-\alpha_{n+1}\left\|x_{n}-p\right\|^{2}
\end{aligned}
$$

which implies that

$$
\begin{aligned}
-\Gamma_{n} & \leq \alpha_{n+1}\left\|x_{n}-p\right\|^{2} \\
& \leq \alpha\left\|x_{n}-p\right\|^{2} \\
& \leq \alpha^{n-n_{0}+1}\left\|x_{n_{0}}-p\right\|^{2}+\frac{\alpha \Gamma_{n_{0}}}{1-\alpha} \\
& \leq\left\|x_{n_{0}}-p\right\|^{2}+\frac{\alpha \Gamma_{n_{0}}}{1-\alpha} .
\end{aligned}
$$

From (3.30) and (3.34), we have

$$
\delta \sum_{n=n_{0}}^{k}\left\|x_{n+1}-x_{n}\right\|^{2} \leq \Gamma_{n_{0}}-\Gamma_{n+1} \leq\left\|x_{n_{0}}-p\right\|^{2}+\frac{\alpha \Gamma_{n_{0}}}{1-\alpha_{0}},
$$

which gives us that $\sum_{n=1}^{\infty}\left\|x_{n+1}-x_{n}\right\|^{2}<+\infty$. Hence

$$
\left\|x_{n+1}-x_{n}\right\| \rightarrow 0
$$

Therefore, $\left\|x_{n+1}-x_{n}\right\| \rightarrow 0$ as $n \rightarrow \infty$. Note

$$
\left\|x_{n+1}-z_{n}\right\|^{2}=\left\|x_{n+1}-x_{n}\right\|^{2}+\alpha_{n}^{2}\left\|x_{n}-x_{n_{1}}\right\|^{2}-2 \alpha_{n}\left\langle x_{n+1}-x_{n}, x_{n}-x_{n-1}\right\rangle .
$$

This implies that $\left\|x_{n+1}-w_{n}\right\| \rightarrow 0$ as $n \rightarrow \infty$. From Lemma 2.7, (3.28) and (3.35), we get

$$
\left\|x_{n}-p\right\|^{2} \rightarrow \ell \text { as } n \rightarrow \infty
$$

From (3.19), we get

$$
\lim _{n \rightarrow \infty}\left\|z_{n}-p\right\|^{2}=\ell
$$

i.e., $\lim _{n \rightarrow \infty}\left\|x_{n}-p\right\|$ exists. Then (3.19) implies that $\lim _{n \rightarrow \infty}\left\|u_{n}-z_{n}\right\|^{2}=\lim _{n \rightarrow \infty}\left\|w_{n}-z_{n}\right\|^{2}=\lim _{n \rightarrow \infty} \| z_{n}-$ $T z_{n} \|^{2}=0$.

Now, we show that the sequence $\left\{x_{n}\right\}$ converges weakly to a point $x^{*} \in \Omega[\operatorname{VI}(C, A)]$. From Proposition 3.3, we have

$$
\left\langle A(x), x-x^{*}\right\rangle \geq 0 \text { for all } x \in C .
$$

It follows, from Lemma $2.6, x^{*} \in \Omega[\mathrm{VI}(C, A)]$.

Theorem 3.5. Let $C$ be a nonempty closed convex subset of a real Hilbert space $X$ and $A: X \rightarrow X$ be a pseudomonotone and L-Lipschitz continuous operator such that $T$ is sequentially weak to weak continuous and $\Omega[\operatorname{VI}(C, A)] \neq \emptyset$. Let $\left\{x_{n}\right\}$ be a sequence in $X$ generated from $x_{1} \in C$ and defined by normal S-iteration based Tseng's extragradient method (3.4), where $\left\{\beta_{n}\right\}$ is a sequence of positive real number satisfying the condition $(D 2)$. Then $\left\{x_{n}\right\}$ be a sequence converges weakly to an element of $\Omega[\operatorname{VI}(C, A)]$.

Proof. The proof follows from Theorem 3.4 by taking $\alpha_{n}=0$ for all $n \in \mathbb{N}$. 


\section{NUMERICAL EXPERIMENTS}

In this section, we provide two numerical examples to test the behaviours of proposed algorithm. Now, we apply Algorithm 3.1 to solve the variational inequality problem and compare numerical results with well-known algorithms. Four algorithms are used to compare here, the Tseng's extragradient method (TEGM) (1.4) and inertial Tseng's extragradient method (ITEGM) (1.5). To show computational effectiveness of the proposed algorithms we use $E_{n}=\left\|x_{n}-x_{n-1}\right\|=10^{-6}$ and $E(x)=\left\|x-P_{C}(x-\lambda A x)\right\|=10^{-6}$ as the stopping criterion.

All the programs are written in Matlab 7.0 and performed on PC Desktop Intel(R) Core(TM) i5-7200U CPU @ $3.18 \mathrm{GHz}, \mathrm{RAM} 8.00 \mathrm{~GB}$. In numerical results reported in the following tables in the number of iterations and the CPU time in seconds. The followings are the example in details.

Example 4.1 ([5]). Suppose $M$ and $N$ be two bounded linear operators on real Hilbert space $X$ into $X$ satisfying $M=M^{*}$,

$$
\langle M x, x\rangle \geq \eta\|x\|^{2} \text { for all } x \in X
$$

where $\eta>0$, and

$$
\langle N x, x\rangle \geq 0 \text { for all } x \in X \text {. }
$$

Let $A: X \rightarrow X$ be defined as

$$
A(x)=\left(e^{-\langle x, M x\rangle}+\alpha\right)(N x+p),
$$

where $\alpha \geq 0$ and $p$ is an arbitrary vector in $X$. Here $A$ is not monotone on $X$ in general. To prove the pseudomonotonocity and Lipschitz continuity of operator $A$ on $X$, we define $f: X \rightarrow \mathbb{R}$ such that $f(x)=e^{-\langle x, M x\rangle}+\alpha$. Let $x, y \in X$ be such that

$$
\langle A(x), y-x\rangle \geq 0 .
$$

Since $f(x)>0$, which implies $\langle N x+p, y-x\rangle \geq 0$. Hence

$$
\begin{aligned}
\langle A(y), y-x\rangle & =f(y)\langle N y+p, y-x\rangle \\
& \geq f(y)(\langle N y+p, y-x\rangle-\langle N x+p, y-x\rangle) \\
& =f(y)\langle N(y-x), y-x\rangle .
\end{aligned}
$$

Now, for every $x, h \in X$ we have

$$
\nabla A(x)(h)=e^{-\langle x, M x\rangle}\langle 2 M x, h\rangle(N x+p)+\left(e^{-\langle x, M x\rangle}+\alpha\right) N h .
$$

Therefore,

$$
\begin{aligned}
\|A(x)\| & \leq 2\|M\| e^{-\langle x, M x\rangle}\left(\|N\|\|x\|^{2}+\|p\|\|x\|\right)+\left(e^{-\langle x, M x\rangle}+\alpha\right)\|N\| \\
& \leq 2\|M\| e^{-\theta\|x\|^{2}}\left(\|N\|\|x\|^{2}+\|p\|\|x\|\right)+\left(e^{-\langle x, M x\rangle}+\alpha\right)\|N\| \\
& \leq L,
\end{aligned}
$$

for some $L>0$, where we took into consideration the fact that the function $t \rightarrow e^{-\eta t^{2}\left(\|N\| t^{2}+\|p\| t\right)}$ is bounded from above on $[0,+\infty]$. This shows that $A$ is pseudo-monotone and Lipschitz continuous on $H$ with constant $L$.

In this case, the feasible set $C$ is a box in $\mathbb{R}^{3}$ defined by $C=\left\{x \in \mathbb{R}^{3}:-5 \leq x_{i} \leq 5, i=1,2,3\right\}$. we take $M=I$, identity matrix of $3 \times 3, N=\left[\begin{array}{ccc}1 & 0 & -1 \\ 0 & 1.5 & 0 \\ -1 & 0 & 2\end{array}\right], \alpha=0.2$ and $p=(-1,2,1)^{T}$ for operator $A$ and iteration parameters are $\mu=\lambda=0.30, \alpha_{n}=0.4$ and $\beta_{n}=0.5$. Take initial point $x_{0}=x_{1}=(1,1,1) \in \mathbb{R}^{3}$. In view of this Figures 1 and 2, we see that Algorithm 3.1 is better than other algorithms mention above (Tab. 1). 


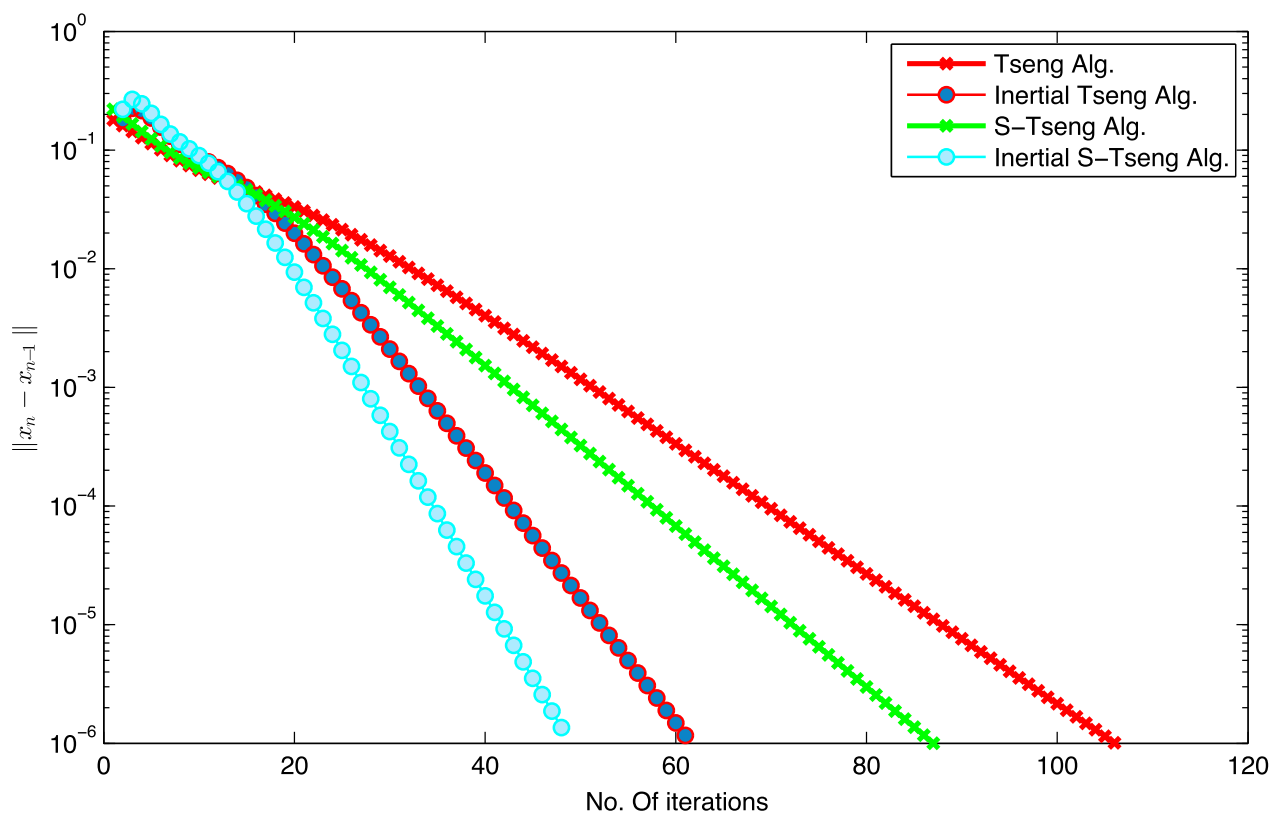

Figure 1. Comparison between no of iteration and $\left\|x_{n+1}-x_{n}\right\|$ for Tseng's Alg. (1.4), InertialTseng's Alg. (1.5), normal S-Tseng's Alg. (3.4) and InS-TEM (3.1).

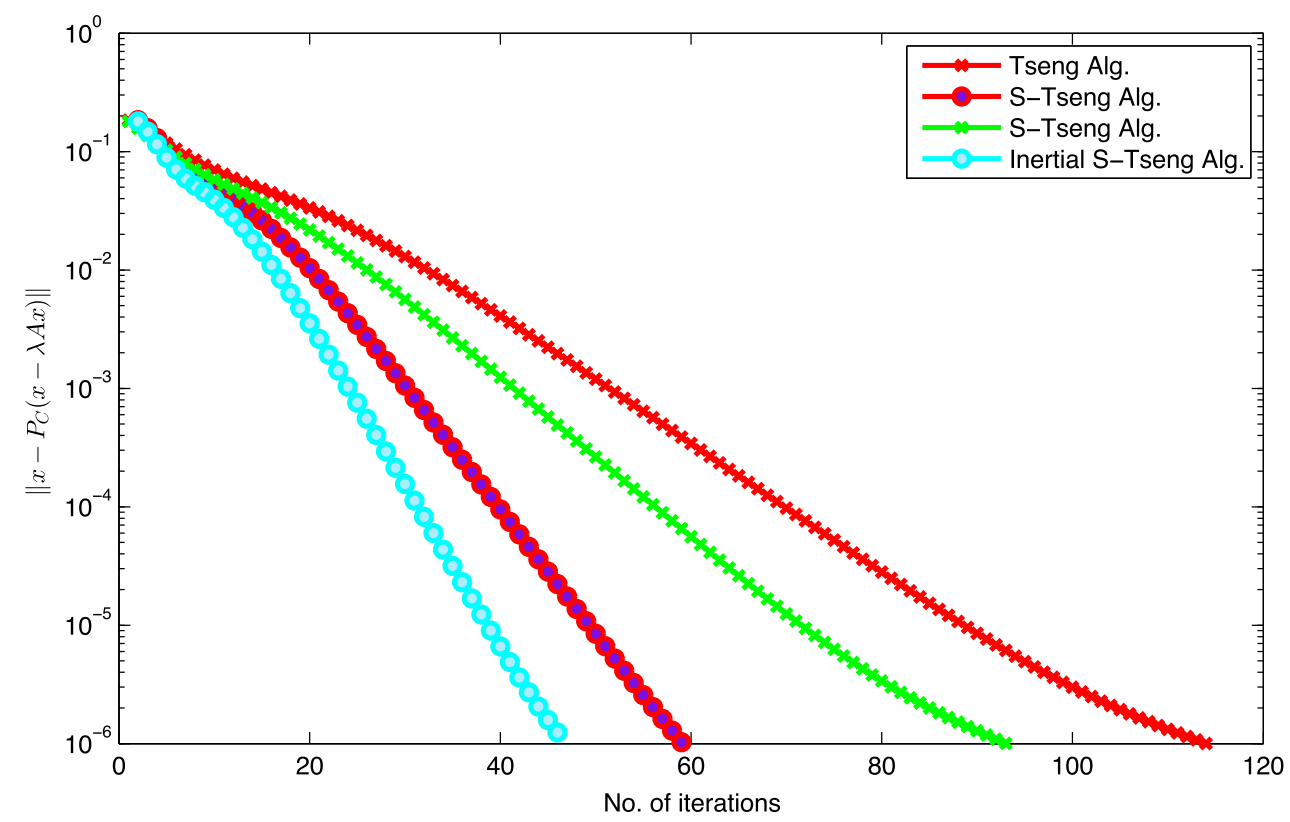

Figure 2. Comparison between no of iteration and $\left\|x_{n+1}-x_{n}\right\|$ for Tseng's Alg. (1.4), InertialTseng's Alg. (1.5), normal S-Tseng's Alg. (3.4) and InS-TEM (3.1). 
TABLE 1. Comparison table in no. of iteration and time for different examples.

\begin{tabular}{|c|c|c|c|c|c|c|}
\hline \multirow[t]{2}{*}{ Example } & \multirow[t]{2}{*}{ Algorithm } & \multirow[t]{2}{*}{ Iter. parameter } & \multicolumn{2}{|c|}{ No. of iteration } & \multicolumn{2}{|c|}{ Time (in seconds) } \\
\hline & & & $\left\|x_{n}-x_{n-1}\right\|$ & $\begin{array}{l}\| x-P_{C} \\
(x-\lambda A x) \|\end{array}$ & $\left\|x_{n}-x_{n-1}\right\|$ & $\begin{array}{l}\| x-P_{C} \\
(x-\lambda A x) \|\end{array}$ \\
\hline EX. (4.1) & Tseng's Alg. (1.4) & $\mu=0.30$ & 107 & 115 & 0.007037 & 0.006304 \\
\hline EX. (4.1) & $\begin{array}{l}\text { Inertial-Tseng's } \\
\text { Alg. (1.5) }\end{array}$ & $\mu=0.30, \alpha=0.4$ & 63 & 60 & 0.008339 & 0.027412 \\
\hline EX. (4.1) & $\begin{array}{l}\text { normal S-Tseng's } \\
\text { Alg. (3.4) }\end{array}$ & $\mu=0.30, \beta=0.5$ & 91 & 94 & 0.010111 & 0.09110 \\
\hline EX. (4.1) & InS-TEM (3.1) & $\begin{array}{l}\mu=0.30 \\
\alpha=0.4, \beta=0.5\end{array}$ & 49 & 47 & 0.008378 & 0.008502 \\
\hline
\end{tabular}

\section{Conclusions}

As we have seen that in Section 1, the normal S-iteration method is an effective method for solving various nonlinear problems. In this paper, we combined normal S-iteration process with inertial Tseng's extragradient method to introduce inertial normal S-iteration Tseng's extragradient method (InS-TEM) for solving the variational inequality problems involving pseudo-monotone and Lipschitz continuous operators. Further, we provide the weak convergence results with the appropriate conditions on iteration parameters and operators. Some numerical example are performed to describe the better accelerating capacity of proposed algorithms.

Acknowledgements. The second author thankfully acknowledges the Council of Scientific and Industrial Research (CSIR), New Delhi, India, through University Grant Commission (UGC) for providing financial assistance in the form of Junior Research Fellowship through grant (Ref. No. 19/06/2016 (i) EU-V)-(434812).

Conflict of interest. The authors declare that they have no conflict of interest.

\section{REFERENCES}

[1] R.P. Agarwal, D. O'Regan and D.R. Sahu, Fixed Point Theory for Lipschitzian-Type Mappings with Applications, Topological Fixed Point Theory and Its Applications. Springer, New York, NY, USA (2009).

[2] F. Alvarez, Weak convergence of a relaxed and inertial hybrid projection-proximal point algorithm for maximal monotone operators in Hilbert space. SIAM J. Optim. 14 (2004) 773-782.

[3] H.H. Bauschke and P.L. Combttes, Convex Analysis and Monotone Operator Theory in Hilbert Space. Springer, Berlin (2011).

[4] R.I. Bot and E.R. Csetnek, An inertial Tsengs type proximal algorithm for nonsmooth and nonconvex optimization problems. J. Optim. Theory Appl. 171 (2015) 600-616.

[5] R.I. Bot, E.R. Csetnek and P.T. Vuong, The Forward-Backward-Forward Method from continuous and discrete perspective for pseudo-monotone variational inequalities in Hilbert spaces. Preprint arXiv:1808.08084 (2018).

[6] X. Cai, G. Gu and B. He, On the $\mathrm{O}(1 / \mathrm{t})$ convergence rate of the projection and contraction methods for variational inequalities with Lipschitz continuous monotone operators. Comput. Optim. Appl. 57 (2014) 339-363.

[7] S.Y. Cho, X. Qin, J.C. Yao and Y. Yao, Viscosity approximation splitting methods for monotone and nonexpansive operators in Hilbert spaces. J. Nonlinear Convex Anal. 19 (2018) 251-264.

[8] A. Dixit, D.R. Sahu, A.K. Singh and T. Som, Application of a new accelerated algorithm to regression problems. Soft Comput. 24 (2020) 1539-1552.

[9] A. Gibali, S. Reich and R. Zalas, Iterative methods for solving variational inequalities in Euclidean space. J. Fixed Point Theory Appl. 17 (2015) 775-811.

[10] A.A. Goldstein, Convex programming in Hilbert spaces. Bull. Am. Math. Soc. 70 (1964) 709-710.

[11] D.V. Hieu and S. Reich, Two Bregman projection methods for solving variational inequalities. Optimization (2020) 1-26. DOI: 10.1080/02331934.2020.1836634.

[12] D.V. Hieu and D.V. Thong, New extragradient-like algorithms for strongly pseudomonotone variational inequalities. J. Global Optim. 70 (2018) 385-399.

[13] D.V. Hieu, Y.J. Cho, Y.B. Xiao and P. Kumam, Modified extragradient method for pseudomonotone variational inequalities in infinite dimensional Hilbert spaces. Vietnam J. Math. (2020) 1-19. DOI: 10.1007/s10013-020-00447-7. 
[14] D.V. Hieu, J.J. Strodiot and L.D. Muu, An explicit extragradient algorithm for solving variational inequalities. J. Optim. Theory App. 185 (2020) 476-503.

[15] D.V. Hieu, Y.J. Cho and Y.B. Xiao, Modified accelerated algorithms for solving variational inequalities. Int. J. Comput. Math. 97 (2020) 2233-2258.

[16] D.V. Hieu, Y.J. Cho, Y.B. Xiao and P. Kumam, Relaxed extragradient algorithm for solving pseudomonotone variational inequalities in Hilbert spaces. Optimization 69 (2020) 2279-2304.

[17] P.D. Khanh, A new extragradient method for strongly pseudomonotone variational inequalities. Numer. Funct. Anal. Optim. 37 (2016) 1131-1143.

[18] P.D. Khanh and P.T. Vuong, Modified projection method for strongly pseudomonotone variational inequalities. J. Global Optim. 58 (2014) 341-350.

[19] D.S. Kim, P.T. Vuong and P.D. Khanh, Qualitative properties of strongly pseudomonotone variational inequalities. Optim. Lett. 10 (2016) 1669-1679.

[20] G.M. Korpelevich, The extragradient method for finding saddle points and other problems. Matecon 12 (1976) $747-756$.

[21] P.E. Mainge and M.L. Gobinddass, Convergence of one-step projected gradient methods for variational inequalities. J. Optim. Theory Appl. 171 (2016) 146-168.

[22] Y.U. Malitsky and V.V. Semenov, A hybrid method without extrapolation step for solving variational inequality problems. $J$. Global Optim. 61 (2015) 193-202.

[23] B.T. Polyak, Some methods of speeding up the convergence of iterarive methods. Zh. Vychisl. Mat. Mat. Fiz. 4 (1964) 1-17.

[24] D.R. Sahu, Applications of the S-iteration process to constrained minimization problems and split feasibility problems. Fixed Point Theory Appl. 12 (2011) 187-204.

[25] D.R. Sahu, Applications of accelerated computational methods for quasi-nonexpansive operators to optimization problems. Soft Comput. 24 (2020) 17887-17911.

[26] D.R. Sahu, A. Pitea and M. Verma, A new iteration technique for nonlinear operators as concerns convex programming and feasibility problems. Numer. Algorithms 83 (2020) 421-449.

[27] D.R. Sahu, J.C. Yao, M. Verma and K.K. Shukla, Convergence rate analysis of proximal gradient methods with applications to composite minimization problems. Optimization 70 (2021) 75-100.

[28] M.V. Solodov and B.F. Svaiter, A new projection method for variational inequality problems. SIAM J. Control Optim. 37 (1999) 765-776.

[29] G. Stampacchia, Forms bilineaires coercitives sur les ensembles convexes. C. R. Acad. Sci. Paris 258 (1964) $4413-4416$.

[30] B.S. Thakur and M. Postolache, Existence and approximation of solutions for generalized extended nonlinear variational inequalities. J. Inequal. Appl. 2013 (2013) 590.

[31] D.V. Thong and D.V. Hieu, An inertial method for solving split common fixed point problems. J. Fixed Point Theory Appl. 19 (2017) 3029-3051.

[32] D.V. Thong and D.V. Hieu, Weak and strong convergence theorems for variational inequality problems. Numer. Algorithms $\mathbf{7 8}$ (2017) 1045-1060.

[33] D.V. Thong and D.V. Hieu, New extragradient methods for solving variational inequality problems and fixed point problems. J. Fixed Point Theory Appl. 20 (2018) 129.

[34] D.V. Thong and D.V. Hieu, Inertial extragradient algorithms for strongly pseudomonotone variational inequalities. J. Comput. Appl. Math. 341 (2018) 80-98.

[35] D.V. Thong and P.T. Vuong, Modified Tseng's extragradient methods for solving pseudomonotone variational inequalities. Optimization 68 (2019) 2203-2222.

[36] P. Tseng, A modified forward-backward splitting method for maximal monotone mappings. SIAM J. Control Optim. 38 (2000) 431-446.

[37] M. Verma and K.K. Shukla, Convergence analysis of accelerated proximal extra-gradient method with applications. Neurocomputing 388 (2020) 288-300.

[38] F.H. Wang and H.K. Xu, Weak and strong convergence theorems for variational inequality and fixed point problems with Tseng's extragradient method. Taiwan. J. Math. 16 (2012) 1125-1136.

[39] Y. Yao, R. Chen and H.K. Xu, Schemes for finding minimum-norm solutions of variational inequalities. Nonlinear Anal. 72 (2010) 3447-3456.

[40] Y. Yao, M. Postolache, Y.C. Liou and J.C. Yao, Construction algorithms for a class of monotone variational inequalities. Optim. Lett. 10 (2016) 1519-1528.

[41] Y. Yao, M. Postolache and J.C. Yao, An iterative algorithm for solving generalized variational inequalities and fixed points problems. Mathematics 7 (2019) 61.

[42] Y. Yao, M. Postolache and J.C. Yao, Iterative algorithms for generalized variational inequalities. UPB Sci. Bull., Ser. A 81 (2019) 3-16.

[43] Y. Yao, M. Postolache and J.C. Yao, Strong convergence of an extragradient algorithm for variational inequality and fixed point problems. U. Politeh. Buch. Ser. A 82 (2020) 3-12.

[44] X.P. Zhao, D.R. Sahu and C.F. Wen, Iterative methods for system of variational inclusions involving accretive operators and applications. Fixed Point Theory 19 (2018) 801-822. 